\title{
Analysis of M2M Capabilities in 4G
}

\author{
J. Poncela $^{\# 1}$, J.M. Moreno $^{\# 2}$, M. Aamir ${ }^{* 3}$ \\ \# Universidad de Málaga, Campus de Excelencia Internacional Andalucía Tech, \\ ETSI Telecomunicación, Malaga, Spain \\ ${ }^{1}$ jponcela@uma.es \\ j jmmorenodic.uma.es \\ * Sir Syed University of Engineering \& Technology and Mehran University of Engineering \& Technology \\ SSUET, Karachi. Pakistan; MUET, Jamshoro, Pakistan \\ muaamir5@yahoo.com
}

\begin{abstract}
M2M (Machine to Machine) communications enable many new applications that reduce the costs of maintenance and operation via remote monitoring and control. The forecasts for this type of communications predict traffic increases associated with these devices of about $100 \%$ in the coming years. However, the behaviour of M2M devices is different from the human user, which causes stress on the networks due to the overload of the signalling procedures. This paper reviews the literature on the current scenario, projections for the decade, and improvements that LTE (Long Term Evolution) will offer for this segment of devices.
\end{abstract}

\section{INTRODUCTION}

3GPP (3rd Generation Partnership Project) defines M2M communications, called MTC (Machine Type Communications) in their standards as "a form of data communication involving one or more entities that do not necessarily require human interaction" ([1], [2]). ITU (International Telecommunication Union) considers M2M as an enabling technology for applications and services in a wide range of vertical markets (e.g. health, logistics, transportation, supplies, etc.). ITU, ETSI (European Telecommunications Standards Institute) and others believe that "a common M2M service layer, agreed at the global level and involving M2M stakeholders and business communities, will provide a cost effective platform" in a multivendor environment [3].

For the IEEE 801.16p working group, Machine to Machine communications are a very different capability that allows the implementation of the "Internet of things". They are defined as the exchange of information between a subscriber station and a server in the core network (via a base station) or between subscriber stations, which can be carried out without any human interaction [2].

The machine type communications are different from existing mobile communication services, as they involve different market scenarios, data communications, a potentially very large number of terminals, and largely little traffic per terminal [1].

M2M devices may connect to servers through a WAN connection (2G-4G) or via M2M gateway, which act as aggregation points [4]. In the latter case, the gateway, being a smart device, collects and processes data received from plain M2M devices and manages their operation. The connection via a gateway is preferable whenever cost, energy consumption or localization have a big impact on the device.
This paper is organized as follows. Section 2 presents forecasts for M2M communications, and the traffic demand required by different scenarios of use. Section 3 comments on the existing capabilities of current networks to support M2M devices. Section 4 describes the limitations in LTE access network signalling, as a prelude to Section 5, where improvements in LTE-A are shown.

\section{M2M SCENARIOS}

According to report [5], the number of M2M connections in the supply industry will increase from 100 million in 2010 to 1.5 billion in 2020 .

The PLC (Power Line Communications) and WiFi mesh technologies will be dominant in this ecosystem. However, although its penetration in 2011 was $83 \%$, in 2020 it will shrink to $67 \%$. This decline will be offset by the cellular networks, which will grow from $13 \%$ to $23 \%$. The same report indicates that $3 \mathrm{G}$ networks, with the initiatives for low cost modules, will overcome from the predominance of $2 \mathrm{G}$ networks in this segment.

A similar forecast is pronounced in [6], which indicates that M2M traffic will multiply by 22 from 2011 to 2016 , representing an average growth of $86 \%$ per year. Network subscriptions of M2M devices will be increased by almost 6 times, from the current 326 million to nearly 2 billion.

In economic and technical terms this is a huge impact for operators, as mobile networks were not designed for this type of communication. In particular, M2M communications have a much lower ARPU (Average Revenue Per User), so costs have to be reduced to ensure profitability. In addition, the expected increase in network devices will bring about a situation of likely overload forcing specific precautions.

M2M communications can cause problematic situations due to, for example, the large number of devices that wish to communicate over a short period of time, such as after a power outage has occurred. This problem has been addressed from two directions [7]: preventive solutions and solutions to manage the network in an overloaded state. In other words, the goal is improving the network so that the probability of an overload is as low as possible, but if it nevertheless comes to happen, using methods such as access restriction may help solve the overload. The network upgrades not always are focused toward situations of overload, but nevertheless they tend to improve the overall network performance. For 
example, techniques to reduce the energy consumption in M2M UEs (User Equipment) may benefit other terminals.

Managing the addressing of large amounts of terminals is another challenge for the network. The IMSI identifier, with only 9 to 10 digits available for MNC identified networks, is the more limiting issue $0 . \mathrm{IPv} 4$ addresses also take their share, but this limitation can be managed deploying NATs (Network Address Translator) and the adoption of IPv6, which provides a larger number of addresses. MSISDN addresses however are not a concern as they may be lengthened to use 20 digits.

For planning, dimensioning and optimizing the network, $\mathrm{M} 2 \mathrm{M}$ also introduces new aspects as the behaviour and localization of M2M devices is different to human users. This means that networks must be planned more from the viewpoint of machine users. As a prerequisite, behaviour, user distributions, etc. of the customer base must be known. This increases the importance of OSS (Operations Support System), BSS (Business Support System) and IT support systems in general as needed entities for generating reports, monitor results, make decisions, and overall, to help manage the complexity of the integral management of customer relationships and maximize $\mathrm{M} 2 \mathrm{M}$ business opportunities.

In UEs, the most difficult issues rely heavily on the scenario of use. In some cases, it may be essential guaranteeing a long duration of batteries, which leads to implementing new optimizations not only in UEs but also on the network.

\section{A. Traffic Demand}

3GPP [1], ETSI [8] and IEEE [2] have addressed the analysis of possible use cases of M2M technology in various environments. For example, 3GPP has identified applications in the areas of security, tracking and tracing, health, remote control, supply counters, etc.. At present the market with the greatest potential for growth in M2M communications infrastructures are supply meters in Smart Grids [9]. Both ETSI and IEEE have defined profiles for this segment. Communication requirements in this scenario vary depending on the application envisaged [10].

The analysis reported in [11], gives an idea of the expected traffic for several types of scenarios of advanced meter infrastructure. In this study, the average message generation rate per meter ranged from $\lambda=1.2 \mathrm{e}-7$ to $\lambda=1.1 \mathrm{e}-4$ with an average message size from $25-30$ bytes up to $2 \mathrm{kB}$ in uplink reports from the meters, but only every 1-2 hours. However, alarm conditions, which may involve a large number of devices, present a challenge to the current network capacity.

Several studies have estimated the number of smart meters by area. For example, in an urban area with a population density of 1 person per $100 \mathrm{~m} 2$, it is estimated between 3,000 and 12,000 meters. Simultaneous access of a substantial part of these smart meters may cause undesirable situations in the network. For example, this can occur as discussed in situations of alarm or power outage, but also if the periodic reports are too frequent. The network must be able to handle a large number of simultaneous non-synchronized access attempts by the deployed devices or implement some mechanism to distribute these accesses on preassigned time slots, while ensuring that the high priority information should not suffer significant delays.

In [2] it is stressed the need for minimal signalling overhead in the connection and data transfer procedures associated with these applications (short communications, medium-high periodicity). It estimates that the overhead for 140 bytes of data ( 53 bytes of net data) is $83 \%$ if only the signalling is considered, but goes up to $220 \%$ if the protocol headers are also considered as overhead.

\section{B. Security}

The security requirements in all previous scenarios are high, as they represent true risks to people and equipment. As stated in [12], the concern is that many M2M applications also open and use both SMS and voice services, and these are rarely encrypted. The question is to what extent M2M applications are dangerous in the hands of authorized third parties. Infrastructure which uses theses services should offer a solution to this problem.

3GPP TR 33.868 [13] standard describes the security features to be met by M2M devices. These include the secure connection between the device and the server, ensuring privacy, the security for small amounts of data and the rejection of messages without integrity protection; improvements in the triggering events, congestion control, time control, monitoring, and restricting the USIM to specific M2M UEs.

In addition, the security services layer [14], includes support for boot credentials, mutual authentication, integrity and confidentiality at the interface with the gateway. The boot procedures can employ TLS (Transport Layer Security), X.509 certificates or EAP (Extensible Authentication Protocol). Along with this, one of the following methods can be used: a) Trust the safety of the access network; b) Use Channel Security (TLS or DTLS - Datagram Transport Layer Security); c) Use object-level security (XML-DSIG and XML-ENC).

\section{CAPABILITIES FOR M2M COMMUNICATIONS}

Nowadays, most M2M applications use GPRS (GSM Packet Radio Service) as the communication technology, employing tools such as the SMS service (Short Message Service) due to their efficiency, provided that the number of devices keeps relatively small [15]. The GPRS air interface traffic resources can be allocated in a static or dynamic way. GPRS allows transporting end-user data as IP packets between. It is designed for bursty applications, like surfing the web or reading email, which is also adapted to M2M applications.

However, GPRS also has drawbacks, which in turn bring about concerns for its appropriateness in M2M applications. For example, the cell capacity relies on parameters like the geographical division used or the reuse pattern. Anyway, if we only take into account the GPRS traffic, the typical spectrum efficiency does not excel 100-150 kb / s / cell / MHz

When any number of voice communications are on the air, the support for data-only users is smaller [16]. It is therefore 
evident that the capacity of GPRS is limited to support foreseen M2M applications and services, where thousands of devices per cell are expected. Another limitation is the need for the connection to be initiated by the device [17].

The capacity constraints in $2 \mathrm{G}$ technologies has been the cause for M2M systems to target other technologies with higher data rates. 3GPP LTE offers this higher radio capacity together with improved management of radio resources (RRM).

From the beginning, LTE has been targeted for broadband applications. But the fact that $\mathrm{M} 2 \mathrm{M}$ applications usually transmit small data quantities, this creates inefficiencies, due to overhead as compared to the net data rate. In addition there are important features, such as the need for low-power devices or lower latencies, which must be taken under consideration. Therefore, the efforts of 3GPP, ETSI and IEEE have been aimed at overcoming the LTE deficiencies for M2M communications [1]. Besides the need to support a large amount of devices, standardisation bodies efforts are also working on supporting a wide range of M2M services, improving energy efficiency, and mapping the coexistence with currently deployed technologies [15].

\section{LIMITATIONS IN LTE SIGNALLING FOR M2M}

In typical scenarios such as those for utilities metering and e- health, the dominant traffic is uplink. As explained in [15], in LTE, the uplink scheduling is performed in the base station eNB (eNodeB) and it indicates the decisions to assign resources to the UE. UEs send their requests through the control signalling channels, i.e. PUCCH (Physical Uplink Control CHannel). PUCCH channels are associated to UEs, so that in the presence of many $\mathrm{M} 2 \mathrm{M}$ devices, as it is foreseen in scenarios such as the previous ones, there may be, at sometimes, a lack of resources on the PUCCH. Furthermore, the channel quality information also occupies resources in uplink control channels. As the number of devices increases, the corresponding signalling load increases too, leading to undesirable performance.

The packet scheduler is the critical mechanism of radio resource management to minimize the overall use of resources while the individual requirements of quality of service QoS [15] are guaranteed. Particularly in LTE, the resource structure in physical blocks (PRB) achieves greater efficiency in the operation of the system. For optimal allocations, the scheduler may take advantage of the channel properties and the dynamic characteristics of the traffic ideally every TTI (Transmission Time Interval). Therefore, uplink and downlink signalling channels, which report the quality information of the channel, the type of traffic and reservations, are necessary to facilitate such scheduling. The features of traffic in M2M scenarios, such as the burst transmissions or the large number of devices significantly increase the complexity and the amount of signalling.

In general, scheduling (as RRM procedure) is not part of the standardization work, but is a particular aspect of each implementation. However, the signalling is standardized, so that within the scope of $3 \mathrm{GPP}$ it has been proposed a general framework for packet scheduling and a set of QoS classes has been defined. Where M2M scenarios appear the QoS criteria are very different. As an example, take into consideration the delay requirements, which may go from several milliseconds (vehicle collision) up to several minutes (smart metering).

The main bottleneck for latency in real-time M2M communications are the access layer procedures [18]. Latency requirements in this layer vary greatly depending on the application, including the delay by DRX (Discontinuous Reception), handovers and HARQ (Hybrid Automatic Repeat Request) mechanisms. The total distribution also depends on the system load and the channel conditions. In LTE, however, the DRX delay can be reduced by suitably selecting the configuration parameters [19], allowing the terminal to wake up in specific time windows.

\section{LTE-A IMPROVEMENTS}

The air interface optimization to efficiently incorporate M2M communications is an area in which the various standards bodies have put considerable effort. For example, GSMA has defined a set of modules specifically for M2M which adapt aspects such as remote management, authentication, air interface communication, thereby reducing costs and, at the same time, it has defined use cases for vertical markets as e-health, automotive, etc.. IEEE has also sought to optimize the 802.16p WiMAX standard, aimed at low power devices, present in large numbers and using burst transmissions. They are also focusing towards cooperative M2M networks [4]. Meanwhile, 3GPP has been releasing improvements in the latest versions:

- Release 10 : It has focused on overload and congestion control. It has also defined a profile for low-priority devices as well as mechanisms to forbid access to devices outside their home or defined network. The congestion control is now also possible in the Access Point.

- Release 11 : Focused on architectural enhancements. Specifically, a new network element and a new control interface have been added. In addition, it supports trigger capabilities, which can be directed towards a specific device service. Includes support for SMS in devices employing only packets regardless of whether they have an MSISDN.

- Release 12 : Improves the management of M2M groups. It is possible to send a single message to a group of devices in a given area. Also, group policies can be defined, for example to limit the maximum group data rate or cut down the number of CDRs (Charging Data Record) that are generated. In addition, it has improvements to for more efficient transmission of single, and small, use data packets on the signalling connection. The main problem is the danger of overloading the connection if the frequency of these transmissions is high (see Fig. 1).

Other improvements in Release 12 are geared towards monitoring, allowing to investigate performance problems by exposing the terminal status and events via 
the Tso interface, the power control optimization, through DRX and long paging cycles, terminal triggering, through signalling rather than SMS, better management of the overhead of these events, and the possibility of cancelling cancel previous requests.

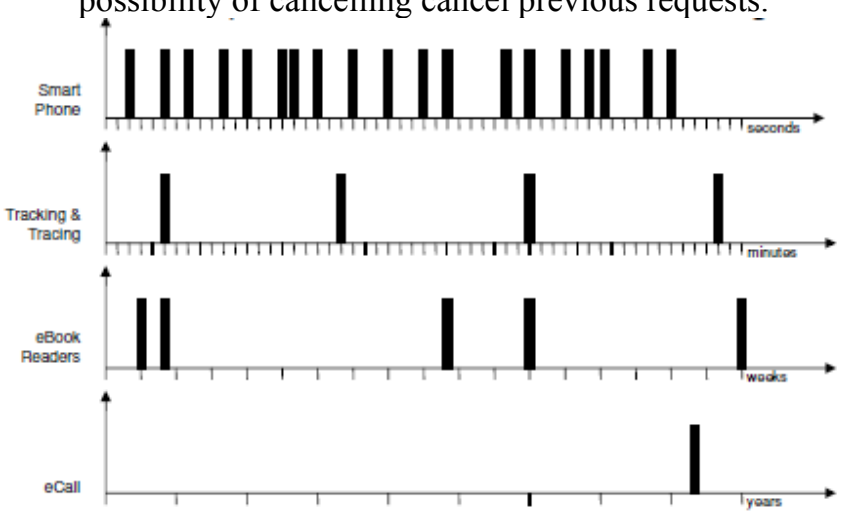

Fig. 1. Transmission pattern for different applications [6]

The architecture defines three types of communication between MTC applications in the UE and MTC applications on servers. These models differ in the manner in which application servers (AS) connect to the 3GPP network and they are designed for a different type of traffic:

- Direct Model. The AS is connected directly to the network to perform operations on the user plane.

- Indirect Model. The AS is connected to the network via a Services Capability Server (SCS) that provides valueadded services. The SCS can belong to the domain of the network operator or the MTC service provider.

- Hybrid model. The other two modes are used simultaneously. It's possible to use SCS in both domains.

\section{Architecture Improvements}

The reference architecture also defines a set of reference points and network elements with specific functionality, previously unavailable, for M2M communications in 3GPP standards.

A new high-level functions named 'Triggering Device' allows the SCS to send information to the UE (usually the execution of some action) even though its IP address is not available or not reachable. The message contains information to route it to the appropriate UE and so that it can forward the response to the application.

\section{Identifiers}

There are two types of identifiers, those defined in general for $3 \mathrm{GPP}$ and those external identifiers. Each MTC subscription has an IMSI, but can store several external identifiers in the HSS. The external identifier must be globally unique and it has two components:

- Domain Identifier. Corresponds with the domain controlled by the mobile network operator and identifies operator services to which access is possible.

- Local ID. Unique in the domain, it is used to obtain the subscription IMSI.
- These identifiers are not exclusive of MTC applications and allow access to terminals without an MSISDN, something that was not possible before.

\section{A. Low-Cost LTE Devices}

It is true that different consumer applications have different requirements, but, according to [20], these are centred on ubiquitous connectivity, cost, ease of use, technological stability and security. LTE is expected to dominate in the near future, with a lifespan similar to that of current consumer equipment. LTE is currently able to meet the requirements of high-end devices, such as TVs, but is an overload for devices such as portable players.

Currently, 3GPP is developing a low-cost version for LTE ecosystem that will enable the consumer electronics to work on a single wireless communications technology. Fig. 2 shows the 3GPP planning to market these products. Upon fruition, LTE for M2M will certainly be a key capability in future consumer electronics.

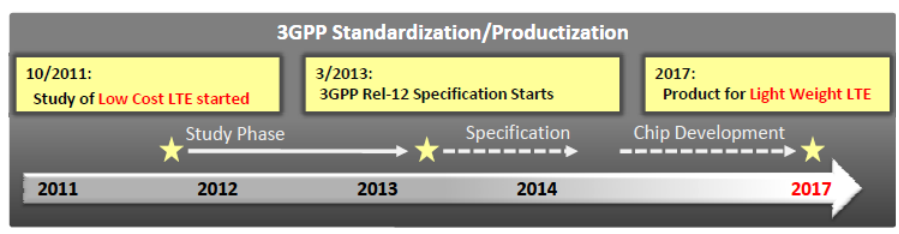

Fig. 2. 3GPP time planning for low-cost LTE devices [20].

The working group RAN1 3GPP is working on possible ways to reduce the cost of $\mathrm{M} 2 \mathrm{M}$ devices, so that it does not hamper the acceptance of the technology by the market. This aspect has already been addressed in some papers like [21], where some requirements have been identified. The low cost LTE devices should operate at data rates which are alike those of a device E-GPRS multi-slot class 2, i.e. $118.4 \mathrm{kbps}$ in the downlink and $59.2 \mathrm{kbps}$ in the uplink.

In [22] and [23] possible ways to reduce the complexity of current LTE devices have been described, including estimates of potential savings. The choices are reducing peak bandwidth, reducing the peak rate or the transmit power, or using the halfduplex mode. Of all these, the main reduction would be using less bandwidth, with an estimated savings of $25 \%$ [24]. The main challenge is to maintain compatibility with devices of Releases 8-10.

\section{B. Radio Access Network}

Two problems posed by M2M communications in current cellular networks are associated with the RAN: signalling overhead and energy consumption in the UE. Some MTC scenarios hold a large number of devices sending application data in relatively small amounts (hundreds of bytes) with periods of several minutes. However, these devices generate a volume similar to the signalling generated by Smartphone terminals, thus occupying network resources inefficiently. Furthermore, many of these devices may have limitations in energy supply (batteries are small or expensive to replace due to geographic inaccessibility), a feature that is not optimized UMTS. 
The signalling overhead can be softened with the use of a management connections optimized by combining RCC messages, definition of a granting procedure for light operation duty and allowing for RCC connections which do not require the establishment of a radio bearer in the $U$-plane [25]. It is also on the table the option of keeping the UE in connected mode.

The solutions studied for the optimization of energy consumption include the use of extended DRX in idle mode, use of long DRX cycles in connected mode, or delaying transmissions until better coverage is found. An analytical study [26] shows that for a given MTC traffic model (small amount of data with fixed time intervals on the order of minutes) the energy savings obtained by the technique of increasing the duration of the DRX cycle is important, reaching $80 \%$ for cycles longer than $25 \mathrm{~s}$ for data intervals of about 15 minutes long. When devices transmit with intervals shorter than $40 \mathrm{~s}$, energy consumption grows very quickly dominated by these transmissions and extending the DRX cycle does achieve savings larger that $20 \%$.

\section{CONCLUSION}

It may be said that for M2M communications to achieve adequate market penetration, the network must optimize interactions with and between $\mathrm{M} 2 \mathrm{M}$ devices taking into account their performance in terms of mobility, frequency and volume of transmissions, alarm signalling, event triggering, group management and safety.

The current LTE standard has a clear limit on the volume of signalling needed to send uplink data as well as in scenarios with massive presence of M2M devices. The improvements proposed in LTE Releases 10-12 tackle these issues, seeking to reduce congestion levels in the case of concurrent access of large number of devices, improve group management, and optimize power consumption.

One of the requirements to make attractive this technology to a wide range of applications is the availability of low cost $\mathrm{M} 2 \mathrm{M}$ devices. This requires acting upon the design of existing equipment, reducing complexity and thus, the need for hardware. Analyzed solutions consist on decreasing the peak data rate, limiting usable bandwidth, simplifying RF modules, or employing half- duplex communications.

The network must support a large number of simultaneous non-synchronized access attempts or have some mechanism to distribute these accesses on preassigned time slots, while ensuring that the high priority information should not suffer significant delays.

\section{ACKNOWLEDGMENT}

This work has been partially funded by Spanish Junta de Andalucía under project TIC-6897, Ingenia (806/39.5949), the Spanish Government (ITC-20111046) and Universidad de Málaga - Campus de Excelencia Internacional Andalucía Tech.

\section{REFERENCES}

[1] 3GPP TS 22.368, "3rd Generation Partnership Project; Technical Specification Group Services and System Aspects; Service requirements for Machine-Type Communications (MTC)", V11.3.0, 2011.

[2] C. HanGyu, "Machine to Machine (M2M) Communications Technical Report", IEEE 802.16 Broadband Wireless Access Working Group, IEEE, 2010.

[3] ITU, "Focus Group on M2M Service Layer", http://www.itu.int/en/ITU-T/focusgroups/m2m/Pages/default.aspx, 2012

[4] G. Wu, S. Talwar, K. Johnsson, N. Himayat, K. D. Johnson, "M2M: From Mobile to Embedded Internet, IEEE Communications Magazine", pp. 36-43, Abril 2011

[5] Machina Research, "Machine-to-Machine (M2M) Communication in the Utilities Sector 2011-20", Machina Report, http://www.giiresearch.com/report/mrl205868-machine-machine-m2mcommunication-utilities-sector.html, 2012.

[6] T. Norp, "Mobile Network Improvements for Machine-Type Communications", ETSI M2M Workshop, 2012.

[7] 3GPP TR 23.888, "System improvements for machine-type communications; (Release 11)", v.1.6.0,, 2011.

[8] ETSI TR 102 691, "Machine-to-Machine communications (M2M); Smart Metering Use Cases", v1.1.1, 2010.

[9] Z. M. Fadlullah, M. M. Fouda, N. Kato, A. Takeuchi, N. Iwasaki, Y. Nozaki, "Towards Intelligent Machine-to-Machine Communications in Smart Grid", IEEE Communications Magazine, vol. 49, no 4, pp. 60-65, 2011.

[10] Department of Energy, "Communications requirements for Smart Grid Technologies",

http://www.smartgrid.gov/sites/default/files/Smart_Grid_Communicati ons_Requirements_Report_10-05-2010.pdf, 2010.

[11] S. Wahle, "Feedback from Implementing the ETSI TC M2M Functional Architecture - Challenges and Opportunities", ETSI TC M2M Workshop, 2012.

[12] J. Cryderman, "How Secure is M2M?", Pipeline, vol. 8, $\mathrm{n}^{\circ} 10$, http://www.pipelinepub.com/0312/OSS_BSS/M2M-Security-3.php, 2012.

[13] 3GPP TR 33.868, "Security aspects of Machine Type Communications", v0.8.0, 2012

[14] ETSI TS 102 690, "Machine-to-Machine communications (M2M); Functional architecture", v1.1.1, 2012.

[15] A. G. Gotsis, A. S. Lioumpas, A. Alexiou, "M2M Scheduling over LTE - Challenges and New Perspectives", IEEE Vehicular Technology Magazine, pp. 34-39, Septiembre 2012.

[16] T. Halonen, J. Romero, J. Melero, "GSM, GPRS and EDGE Performance: Evolution Towards 3G/UMTS", Wiley, 2002.

[17] M. Martsola, T. Kiravuo, J. Lindqvist, "Machine to machine communication in cellular networks", Int. Conf. Mobile Technology, Applications and Systems, pp. 6, 2005.

[18] N. Nikaein, S. Krco, "Latency for Real-Time Machine-to-Machine Communication in LTE-Based System Architecture", European Wireless, pp. 263-268, 2011.

[19] C.S. Bontu, E. Illidge, "DRX mechanisms for power saving in LTE", IEEE Communication Magazine, vol. 47, $\mathrm{n}^{\circ}$ 6, pp. 48-55, 2009.

[20] Y. Morioka, "Low Cost LTE for M2M Consumer Electronics", ETSI TC M2M Workshop, 2012.

[21] Vodafone et al., RP-111112, "Provision of low-cost MTC UEs based on LTE", RAN plenary \# 53, 2011.

[22] R1-113440, "Initial complexity analysis of MTC LTE UEs", RAN1 \# 66bis, 2011.

[23] M. Beale and Y. Morioka, "Support of Low Complexity LTE Terminals". IEEE VTC 2012 Spring conference, pp. 1-6, 2012

[24] M. Beale, "Future Challenges in Efficiently Supporting M2M in the LTE Standards", WCNC 2012 Workshop on Internet of Things Enabling Technologies, Embracing Machine-to-Machine Communications and Beyond, pp. 186-190, 2012.

[25] 3GPP TR 23.887 "Machine-Type Communication and other mobile data applications communications enhancements"”, (Release 12), V1.3.0, 2013

[26] T. Tirronen, A. Larmo, J. Sachs, B. Lindoff, N. Wiberg, "Reducing energy consumption of LTE devices for machine-to-machine communication”. IEEE Globecom 2012. 